
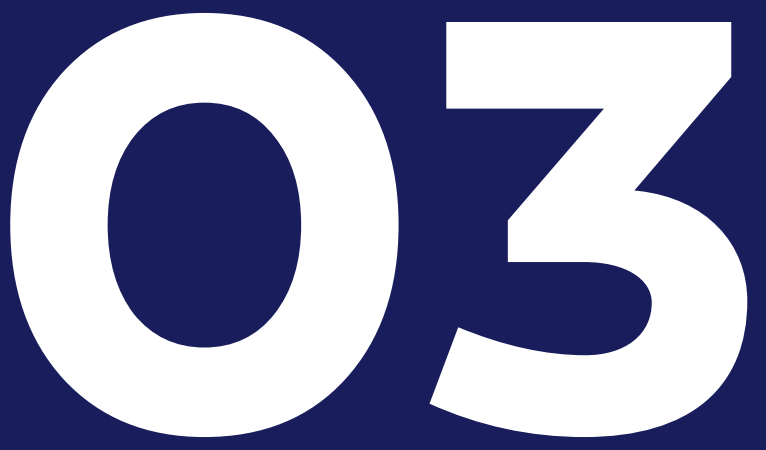

\title{
Análisis con datos de panel de los factores determinantes del nivel de endeudamiento de las grandes empresas ecuatorianas del sector manufacturero
}

Analysis with panel data of the determinig factors of the level of indebtebness or Ecuadorian large manufacturing companies

FECHA DE RECEPCIÓN: 07/12/2020

FECHA DE APROBACIÓN: 05/03/2021

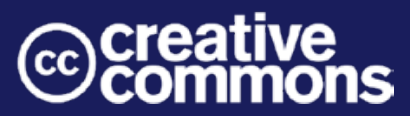




\section{Luis Gabriel Pinos Luzuriaga ${ }^{1}$ Wazhington Bladimir Proaño Rivera ${ }^{2}$ Luis Bernardo Tonon Ordóñez ${ }^{3}$ Silvia Raquel Mejía Matute ${ }^{4}$}

La política de financiación establece cómo deberán ser obtenidos los recursos que cubrirán las necesidades de fondos de la empresa. El objetivo de este estudio fue determinar las variables que explican el nivel de endeudamiento de 218 grandes empresas del sector manufacturero ecuatoriano, durante el período 2008-2018 y qué teoría ajusta de mejor manera las decisiones de financiamiento de estas empresas, así como comparar los resultados obtenidos con los propuestos por las teorías de trade offy jerarquía financiera. La metodología empleada fue una estructura de datos de panel, con un modelo de efectos fijos; se realizó la estimación a través de mínimos cuadrados ordinarios lo que permitió conocer la significancia global del modelo, así como las variables que explican el nivel de endeudamiento y su respectiva significancia estadística. Las variables que resultaron ser estadísticamente significativas para explicar el nivel de endeudamiento de las empresas fueron: tamaño, reputación y liquidez. En este estudio la teoría de jerarquía financiera tuvo mayor poder explicativo a la hora de evaluar la forma de cómo se financian las empresas grandes del sector manufacturero ecuatoriano.

Palabras Clave: Datos de panel, endeudamiento, estructura de capital, jerarquía financiera, trade off

\footnotetext{
1 U. del Azuay · Ipinos@uazuay.edu.ec • https://orcid.org/0000-0002-3894-8652

2 U. del Azuay · wproano@uazuay.edu.ec · https://orcid.org/0000-0003-4959-7410

3 U. del Azuay · Itonon@uazuay.edu.ec · https://orcid.org/0000-0003-4959-7410

4 U. del Azuay · smejia@uazuay.edu.ec · https://orcid.org/0000-0003-1256-8165
} 


\section{Abstract}

The financing policy establishes how the resources that will cover the company's funding needs should be obtained. The objective of this study was to determine the variables that explain the level of indebtedness of 218 large companies in the Ecuadorian manufacturing sector, during the period 2008-2018, and which theory best adjusts the financing decisions of these companies, as well as to compare the Results obtained with those proposed by the theories of trade off and financial hierarchy. The methodology used was a panel data structure, with a fixed effects model, the estimation was carried out through ordinary least squares, which allowed knowing the global significance of the model, as well as the variables that explain the level of indebtedness and its respective statistical significance. The variables that turned out to be statistically significant to explain the level of indebtedness of the companies were: size, reputation and liquidity. In this study, the financial hierarchy theory had greater explanatory power when evaluating the way in which large companies in the Ecuadorian manufacturing sector are financed.

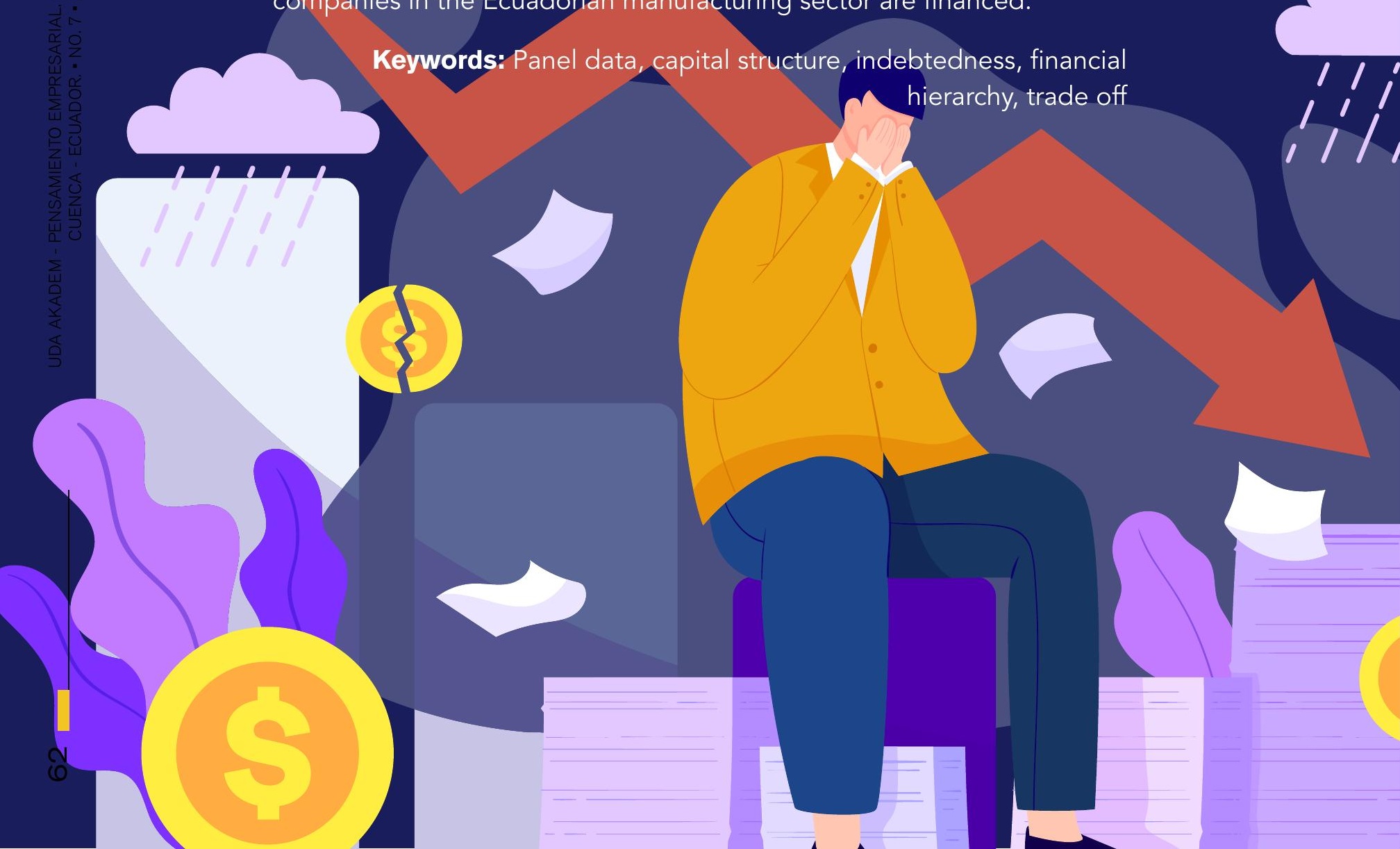




\section{Introducción}

no de los principales factores de crecimiento de cualquier empresa, pública o privada, es la adquisición de activos para incrementar sus capacidades productivas. En este orden de ideas se hace necesario contar con capital para tal fin. A lo largo del estudio, se analizaron las diferentes teorías (relevancia e irrelevancia de una estructura de capital), que tratan de explicar la forma cómo las empresas buscan financiamiento.

Las empresas pueden recurrir a diversos mecanismos de financiamiento: fuentes internas, como son las utilidades retenidas; $y$, fuentes externas, como son el endeudamiento y la emisión de acciones. Decidir una combinación de deuda con recursos propios es una decisión difícil, pues, el alto nivel de deuda puede elevar la probabilidad de quiebra de las empresas, pero, simultáneamente, reduce el pago de impuestos debido al ahorro fiscal. Por otra parte, un bajo nivel de endeudamiento puede suponer una reducción de la rentabilidad a causa de mayor presión fiscal, que conlleva problemas de sobre inversión y reducción de riqueza para los accionistas (Gutiérrez et al., 2018).

Tal como lo expone Rivera (2002), a lo largo de la historia han aparecido teorías que tratan de explicar la forma cómo las empresas financian sus inversiones. Por un lado, se tienen las teorías que explican la relevancia de la estructura de capital como, por ejemplo, los estudios de Durand (1952) y Schwartz (1959) pasando por Modigliani y Miller (1963); luego, con la teoría de la agencia de Kim y Sorensen (1986), Jensen (1986), Stulz (1990) y llegando, finalmente, a una teoría que resume la relevancia de la estructura de capital llamada trade off. Por otro lado, están las teorías que hablan sobre la irrelevancia de la estructura de capital; entre sus principales exponentes citamos: Modigliani y Miller (1958); y Miller (1977) llegando, por último, al principal referente de la teoría de irrelevancia que es Myers (1984) quien fundamenta la teoría de la jerarquía financiera o pecking order. 
A partir de estos estudios, se han desarrollado un sin número de análisis en Latinoamérica, teniendo como ejemplo los trabajos de Wadnipar y Cruz (2003); Mongrut, Fuenzalida, Pezo y Zdenko (2010); Medina, Salinas, Ochoa y Molina (2012); Padilla, Rivera y Ospina (2015); Paredes, Ángeles y Flores (2015) y el de Contreras y Díaz, (2015). En el caso de Ecuador, existen pocos trabajos entre los que se destacan: Gutiérrez, Moran y Posas (2018) y Montalván (2019), en donde los resultados son variados a la hora de explicar las teorías que mayor ajuste tienen.

Si se contemplan las empresas en Latinoamérica y sobre todo en Ecuador, los estudios demuestran que, en promedio, la generación interna de fondos (utilidades más la depreciación), cubre la mayor parte del efectivo que necesitan las empresas para realizar sus inversiones, por lo que parecería que el financiamiento interno es preferido al financiamiento externo, sobre todo, el proveniente con emisión de acciones o de deuda, siendo que los administradores tengan un grado de aversión irracional o egoísta hacia el financiamiento externo. Un administrador que quiera tener un empleo cómodo, puede verse tentado a abandonar un proyecto riesgoso, aunque tenga un valor actual neto positivo, si esto implica el lanzamiento de una nueva emisión de acciones o deuda y tener que enfrentar preguntas incómodas por parte de los inversionistas. Tal vez los administradores tomen una línea de resistencia mínima y eviten la disciplina del mercado de capital (Brealey, Myers y Allen, 2015). 


\section{Métodos}

\subsection{Revisión de literatura}

Las empresas invierten en activos de corto y largo plazo, con fondos que se obtienen de dos maneras: primero, a través de fondos generados internamente como beneficios retenidos, pero, a veces resultan insuficientes para cubrir sus necesidades de inversión y se genera un déficit de financiamiento, por lo que se opta por el segundo tipo de alternativa de financiamiento; es decir, utilizar fondos externos como la emisión de acciones o el endeudamiento.

Lo expuesto anteriormente plantea las siguientes interrogantes: ¿Qué parte de los beneficios debe retenerse? y ¿Cuánto del dinero que la empresa necesita debe ser financiado con endeudamiento en lugar de emisión de acciones? Para responder la primera pregunta, la empresa debe definir una política de dividendos, esto quiere decir que deberá decidir entre el porcentaje de retención de utilidades y la razón de pago de dividendos; mientras que, para responder a la segunda pregunta, la empresa necesitará una política de endeudamiento (Brealey, Myers y Allen, 2015). En el presente trabajo se determinarán los factores que explican al nivel de endeudamiento de las grandes empresas manufactureras en Ecuador, por lo tanto, para realizar una validación empírica sobre los factores de endeudamiento de una empresa, se hace necesario determinar los conceptos que afectan al endeudamiento empresarial y, por tanto, a su estructura de capital.

Se define a la deuda como un préstamo a una empresa, gobierno o individuo (Besley y Brigham, 2016) y esta tiene tres características esenciales: el monto de capital que debe pagarse, los pagos de los intereses y la fecha de vencimiento. Toda empresa tiene dos tipos de financiamiento vía deuda: deuda de corto plazo y deuda de largo plazo; la primera, se refiere a fondos con vencimiento menor o igual a un año; mientras que, la segunda, son los fondos con vencimiento mayor a un año. 
Un concepto muy relacionado con el endeudamiento es el apalancamiento financiero que Besley y Brigham (2015) lo definen como un cambio porcentual en las utilidades por acción, debido a cambios porcentuales en la utilidad antes de intereses e impuestos. El apalancamiento financiero surge por la existencia de costos fijos en los que se incurren con el endeudamiento y, por lo tanto, están relacionados con la forma de financiar a la empresa. Es decir, cuanta mayor dificultad tenga una empresa de pagar sus costos fijos financieros con su utilidad antes de intereses e impuestos, más alto será su grado de apalancamiento financiero y, por tanto, se consideran empresas más riesgosas que las que tienen un menor grado de apalancamiento financiero.

La importancia de estudiar la estructura de capital en una empresa radica en la generación de modelos que permitan determinar la estructura 'óptima', para hacer uso eficiente de los recursos (Gutiérrez et al., 2018), para lo cual, las empresas deben definir una política de estructura de capital, lo cual implica una compensación entre riesgo asumido y rendimiento generado (Besley y Brigham, 2016).

El debate sobre este tema comenzó con la postura tradicional de relevancia de la estructura de capital en el valor de la empresa estudiado por algunos autores, entre los que destacan están Durand (1952) y Schwartz (1959), cuyas propuestas están basadas en la existencia de una estructura financiera óptima, con uso de la deuda como fuente de financiamiento, ya que es más barata debido a que los acreedores asumen menos riesgo que los tenedores de acciones; $y$, el rendimiento requerido por los acreedores será menor al rendimiento requerido por los tenedores de acciones, por lo tanto, disminuirá el costo promedio ponderado de capital.

Por su parte, Modigliani y Miller (1958) demostraron que en mercados perfectos de capitales las decisiones de la estructura financiera son irrelevantes. Un mercado perfecto de capitales se refiere a que: 1) no existen impuestos, 2) no existen costos relacionados con la quiebra, y 3) no existen costos de la agencia y asimetrías de la información. Los supuestos mencionados anteriormente soportan la hipótesis de que el valor de la empresa no está influido por el instrumento de financiamiento escogido, además, que el valor de la empresa está basado en sus activos reales y no en sus títulos emitidos; y, finalmente, que el valor de una empresa sin deuda es el mismo al de una empresa con deuda. Hoy en día 
se sabe que estos supuestos no son reales, pero, el aporte que dieron los autores fue iniciar la discusión sobre la investigación formal de las finanzas corporativas. Años después, los mismos autores reconocieron las fallas del estudio publicado en 1958, añadiendo las ventajas fiscales por el uso de la deuda ya que generan intereses que a su vez son deducibles del pago de impuestos; por tanto, argumentaron que los impuestos influyen sobre la estructura de capital de la empresa, pero que, a pesar de las ventajas del endeudamiento, las empresas no deberían incurrir en la cantidad máxima de deuda potencialmente garantizada por sus activos, pues, elevaría la probabilidad de quiebra (Modigliani y Miller, 1963).

Miller (1977) se pronunció sobre lo difícil que es encontrar un equilibrio entre los beneficios de la deuda y los costos de quiebra, llegando a la conclusión de que la ventaja de la deuda se elimina en un mundo de aumento progresivo de impuestos, eliminando los incentivos de emitir más deuda y retomó la tesis de irrelevancia de la estructura de capital sobre el valor de la empresa.

Posteriormente, Kim y Sorensen (1986) plantean la teoría de costos de agencia y mencionan que la estructura de capital de la empresa se establece mediante una serie de contratos entre diferentes grupos de interés (principal, agente y acreedor) en donde el accionista (el principal) de la empresa da autoridad a otra persona para que gestione la empresa a su nombre (el agente). El problema surge cuando los intereses del principal y del agente no están alineados y generan los problemas de la agencia. Autores como, Jensen (1986) y Stulz (1990) indican que los problemas de la agencia tienden a reducirse con el uso de la deuda. Cuando los gerentes no están alineados con los intereses de los accionistas, hay un problema de flujo de caja libre; es decir, cuando la empresa genera flujo de caja superior al que necesita para financiar sus proyectos de inversión, el accionista querrá que ese excedente se convierta en dividendos; mientras que, el gerente querrá transformarlos en beneficios personales, por este motivo las empresas emiten deuda para reducir la discrecionalidad de los gerentes a través del pago de los intereses y restringiendo inversiones de alto riesgo. La razón de este hecho se debe a que el pago de los intereses y capital actúa como un mecanismo controlador, de tal manera, que se estaría limitando el dinero para desembolsos estrictamente necesarios. 
La teoría del trade off o de equilibrio de la estructura de capital resume todas las teorías y modelos que proponen una estructura de capital óptima, que maximiza el valor de la empresa que se obtiene a partir de un equilibrio entre los costos (quiebra y agencia) y beneficios fiscales (escudo tributario) de la deuda (Zambrano y Acuña, 2011). Además, establece un límite al endeudamiento propuesto por Miller y Modigliani (1963). El trade off justifica proporciones moderadas de endeudamiento; es decir, la empresa se endeudará hasta el punto en donde el valor marginal del escudo de impuestos en deuda adicional se ve disminuido por el valor actual de los posibles costos de quiebra o reorganización y los costos de la agencia, que se incrementan cuando existe duda sobre la credibilidad de la deuda de la empresa (Wadnipar y Cruz, 2008).

Por último, la teoría de jerarquía de preferencias o pecking order, supone asimetría de información, por lo que los administradores saben más de los prospectos, riesgos y valores de la compañía que los inversionistas externos (Brealey, Myers y Allen, 2015). La teoría del pecking order establece que no existe una estructura óptima de capital que equilibre los beneficios y costos de la deuda, sino más bien, los gerentes incrementan sus inversiones usando una jerarquía de preferencias en cuanto al uso de fuentes de financiación; es decir, siguen una escala de jerarquías a la hora de buscar financiación recurriendo, primero, a financiación interna (utilidades retenidas) y, luego, a financiación externa priorizando la deuda y, posteriormente, a la emisión de acciones.

El principal referente de la teoría de jerarquía de preferencias fue Myers (1984), quien menciona que las empresas prefieren financiarse con recursos internos, es decir, con utilidades retenidas para aprovechar las oportunidades de inversión con el fin de evitarse los costos relacionados por la información asimétrica del mercado.

De los muchos estudios realizados sobre esta teoría se puede destacar el de Andrés, De la Fuente y San Martín (2018), que por medio de una encuesta realizada a 140 grandes empresas españolas se concluyó que los directivos encuestados prefirieron el financiamiento interno al externo y, en caso de acudir a este este último, el financiamiento bancario el más importante. 
Para el caso latinoamericano, hay la experiencia de estudios previos para determinar la estructura de capital en las empresas; podemos citar a Wadnipar y Cruz (2003), el motivo del estudio fue encontrar si en la racionalidad del accionista colombiano está presente la jerarquización de sus fuentes de financiamiento o se inclinan hacia la teoría de trade off, concluyendo que las empresas colombianas se ajustaron a un modelo pecking order o jerarquización de las fuentes de financiamiento y que esta teoría se ajusta en un nivel semi fuerte, ya que la variable déficit no fue la única que explica el nivel de cambio de la deuda adquirida, sino también variables como: oportunidades de crecimiento, tamaño de la empresa y tangibilidad de los activos.

En Ecuador se han presentado algunos estudios sobre la estructura de capital de las empresas; Montalván (2019) que aplicó un modelo de datos de panel a información de 63 Pymes con financiamiento en el mercado de valores. Las variables que resultaron ser significativas para explicar la estructura de capital de las empresas fueron: tamaño, oportunidades de crecimiento y escudo fiscal; mientras que, la rentabilidad y el valor del colateral no fueron relevantes. Si la empresa crece o aumenta su tamaño, la inclinación por el financiamiento con deuda crece; mientras que, el escudo fiscal a pesar de no ser una variable tan robusta, tiene un efecto positivo en la del aumento de la deuda.

Por otro lado, Gutiérrez, Morán y Posas (2019) trataron de explicar las variables que determinan la estructura de capital en 304 empresas del sector manufacturero de la ciudad de Guayaquil, entre el período 2012 y 2016, por lo que para el análisis plantearon un modelo de datos de panel de efectos fijos, siendo la teoría de jerarquía financiera la que tuvo mayor poder explicativo que la de equilibrio estático; además, no se encontró evidencia que sustente la importancia de las expectativas de crecimiento sobre la estructura de capital. 


\subsection{Metodología}

Se planteó un estudio exploratorio, empírico y cuantitativo sobre las grandes empresas del sector manufacturero (sector C) del Ecuador, durante el período 2008-2018. La población objeto de estudio, son las empresas que se han mantenido activas durante este período y que han reportado su información financiera ante la Superintendencia de Compañías, Valores y Seguros (2020). En total fueron 218 clasificadas según CIIU 4.0; se eliminó de la base de datos a empresas que no disponían, en algunos de los años en estudio, de la información para la realización del análisis. Para la elaboración del modelo econométrico se utilizó el software Eviews 10.0.

Para estimar los determinantes del endeudamiento de las empresas del sector, se procedió a la aplicación de un modelo con datos de panel o longitudinales (Fernández y Murillo, 2014). Gujarati y Porter (2010) mencionan que al referirse a datos de panel se combinan datos de sección cruzada (corte transversal) con datos de series de tiempo.

Según Grenne (2000), los datos de panel ofrecen un entorno muy rico para el desarrollo de técnicas de estimación y resultados teóricos. Sin embargo y desde el punto de vista más práctico, los investigadores han podido utilizar datos de series temporales y de sección cruzada para examinar cuestiones que no podrían estudiarse en contextos de series temporales o sección cruzada por sí solos.

En la investigación económica y financiera se trabaja con datos de panel que contienen observaciones de países, industrias $y$, en este caso, empresas observadas en el tiempo. Por tanto, se utiliza esta técnica cuando hay $\mathrm{N}$ unidades individuales (218) distintas, observadas en $\mathrm{T}$ diferentes períodos (11). 
- Al estudiar empresas a lo largo del tiempo, lo más seguro es encontrar heterogeneidad en las unidades, por lo que la técnica de datos de panel toma en cuenta dicha heterogeneidad al permitir la creación de variables específicas para cada nivel de corte transversal.

- Proporcionan más información, más grados de libertad, menos colinealidad entre variables y mayor eficiencia.

- Detectan y miden mejor los efectos que no se puede observar en datos puramente de corte transversal o serie de tiempo (Gujarati y Porter, 2010).

Para este estudio, hay 218 unidades de corte transversal (empresas) observadas desde el año 2008 al 2018. Se trabajó con un panel balanceado y corto; se lo denomina de esta manera por cuanto el número de unidades de corte transversal es mayor que el número de períodos.

Según Gujarati y Porter (2010) existen cuatro posibles modelos que se pueden trabajar con datos de panel balanceado:

" Modelo Agrupado de MCO (MCG).

- Modelo de mínimos cuadrados con variable dicótoma de efecto fijo (MCVD).

- Modelo de efectos fijos dentro del grupo (DG).

- Modelo de efectos aleatorios (MEFA).

El modelo agrupado de $\mathrm{MCO}$ se estima a través de una gran regresión al hacer una agrupación de las 2180 observaciones (218*10), sin entender la naturaleza de corte transversal y serie de tiempo; por lo tan- 
to, se oculta la heterogeneidad o singularidad que pueden existir entre las diferentes empresas.

El modelo de mínimos cuadrados con variable dicótoma de efecto fijo (MCVD) al permitir que cada empresa tenga su propio valor de intercepto, toma en cuenta la heterogeneidad entre las diferentes empresas, el intercepto es invariante en el tiempo $\left(b_{1 i}\right)$, su especificación es:

$$
Y_{i t}=B_{1 i}+b_{2} X_{1 i t}+b_{3} X_{2 i t}+u_{i t}
$$

A diferencia de la regresión agrupada que sesga la estimación de la pendiente, el modelo con variable dicótoma de efectos fijos permite que el intercepto varíe entre empresas, por lo que usa variables dicótomas con intercepto diferencial que se expresa de la siguiente manera:

$$
Y_{i t}=\alpha_{1}+\alpha_{2} D_{2 i}+\alpha_{3} D_{3 i}+\ldots+\alpha_{n} D_{n i}+\beta_{2} X_{1 i t}+\beta_{3} X_{2 i t}+u_{i t}
$$

Donde

$D_{2 i}=1$ si la observación corresponde a la empresa 2 y 0 en otro caso.

$D_{3 i}=1$ si la observación corresponde a la empresa 3 y 0 en otro caso.

Se debe recordar que es necesario evitar la trampa de la variable dicótoma, por lo que es necesario tener n-1 unidades de corte transversal, siendo la empresa uno la categoría base; en todo caso, si quiere introducir la variable dicótoma para cada empresa es necesario obviar el intercepto. 
Existen algunos problemas con el trabajo del MCVD de efectos fijos:

- Problema de los grados de libertad al introducir muchas variables dicótomas.

- Aumenta la posibilidad de multicolinealidad.

- No identifica el efecto de las variables que no cambian en el tiempo.

Por otro lado, el modelo de efectos fijos dentro del grupo, elimina el efecto fijo $B_{1 i^{\prime}}$ expresando los valores de las variables dependientes e independientes de cada empresa como desviaciones de sus respectivos valores medios. La diferencia con las dos anteriores es que la primera omite la heterogeneidad de las empresas; mientras que, esta la toma en cuenta, no por el método de la variable dicótoma, sino que la elimina mediante la diferenciación de las observaciones de la muestra en torno a sus medias muestrales.

En cambio, en el método de efectos aleatorios al especificar el modelo de regresión, no se pueden incluir variables explicativas relevantes que no varíen en el tiempo y, posiblemente, otras que sí varían en el tiempo, pero, tienen el mismo valor en todas las unidades de corte transversal, la inclusión de variables dicótomas es un encubrimiento de nuestra ignorancia (Gujarati y Porter, 2010).

Por lo tanto, si la aplicación de las variables dicótomas refleja la falta de conocimiento del modelo, en el modelo de efectos aleatorios, incorpora esta ignorancia en el término del error.

Si hay heterogeneidad inobservable en un sistema de datos de panel a través del tiempo o entre las unidades de estudio (en nuestro caso empresas) o en ambos, hay que buscar una especificación que capture estos rasgos para evitar sesgos de especificación. Para dar solución a este problema existen los modelos de efectos fijos y aleatorios (Baronio y Vianco, 2014). 
El modelo de efectos fijos permite una correlación arbitraria entre el efecto fijo o inobservable con la variable regresora; mientras que, el modelo de efectos aleatorios no. Adicionalmente, si la variable explicativa clave es constante en el tiempo, no es posible usar efectos fijos para estimar el efecto sobre la variable dependiente (Wooldridge, 2010).

Para la determinación del uso de modelos de efectos fijos o aleatorios, Gujarati y Porter (2010), proponen la utilización del test de Hausman; en esta prueba, la hipótesis nula es que los estimadores de efectos fijos y aleatorios no varían considerablemente, de tal manera que, al rechazar la hipótesis nula se dirá que el método de efectos aleatorios no es el adecuado, ya que es probable que los efectos aleatorios estén correlacionados con una o más regresoras.

Mayorga y Muñoz (2000) mencionan que la elección entre efectos fijos o aleatorios depende de: a) El objetivo del estudio, b) el contexto de los datos, y c) número de datos disponibles. En el primer caso, los autores mencionan que si se trabaja con una muestra aleatoria, lo mejor será trabajar con un modelo de efectos aleatorios; mientras que, si la muestra es seleccionada a conveniencia o se trabaja con datos poblacionales, el modelo de efectos fijos será el mejor. En el segundo, mencionan que con el modelo de efectos fijos, la heterogeneidad no observable se incorpora en la ordenada al origen; mientras que, en los efectos aleatorios en el error, por lo que se modifica la varianza del modelo. Los modelos de efectos fijos y de efectos aleatorios tienen diferencias si se generan con un $t$ pequeño y un $N$ grande. Este criterio está muy relacionado con la tercera razón: si tenemos un $N$ grande y t pequeño, podría ser que el número de parámetros de los efectos fijos sea muy grande en relación al número de datos obtenidos, dando estimaciones ineficientes.

Una vez definida claramente la estrategia econométrica se definen las variables a utilizar en el estudio.

La variable dependiente es el nivel de endeudamiento, que se define como la razón deuda total sobre activo total:

$$
\text { Nivel de endeudamiento }=\frac{\text { Deuda Total }}{\text { Activo Total }}
$$


Como se evidencia no se diferencia entre deuda de corto plazo y largo plazo.

Las variables que explican el nivel de endeudamiento son:

Tamaño de la compañía: el tamaño es un factor explicativo importante a la hora de evaluar el endeudamiento, ya que de él depende la capacidad de endeudamiento y respaldo existente en la empresa. Este valor se encuentra en función de los activos totales y es aquella con mayor cantidad de activos la empresa que mayor respaldo podrá ofrecer. El tamaño de la empresa también se puede valorar observando los ingresos.

$$
\text { Tamaño= Ln (ingresos) }
$$

Tamaño $=\operatorname{Ln}$ (activo Total)

Al usar logaritmos, disminuyen los niveles de las series de tiempo.

La teoría del trade off predice una relación positiva entre el tamaño y el nivel de endeudamiento, la razón es porque la empresa más grande es mucho más diversificada que la empresa más pequeña y con esto su probabilidad de quiebra es menor; así también, el tamaño es un importante mecanismo para tener poder de negociación con acreedores. Autores como Fernández y Murillo (2014); Acedo, Ulutiz y Ruiz (2012); Serrasqueiro y Caetano (2015) coinciden que hay una relación positiva y estadísticamente significativa entre el tamaño de la empresa y el nivel de endeudamiento. 
Por otro lado, la teoría de jerarquía de preferencias, predice una relación negativa entre el tamaño y el nivel de endeudamiento, con lo cual, se priorizarán fuentes de financiamiento internas como utilidades retenidas para financiar su crecimiento. Autores como Acaravci (2015); Paredes, Ángeles y Flores (2015), han obtenido una relación negativa entre el endeudamiento y el tamaño empresarial. Dentro de los autores que han demostrado que la variable tamaño empresarial es estadísticamente no significativa está Tresierra (2008).

Garantías: las garantías serán el ofrecimiento que la compañía puede hacer como respaldo real a los inversionistas o prestamistas, pues, serán estos los elementos prendarios o a hipotecarse para los prestamistas y los elementos prioritarios. Estas se calculan como el valor de los activos netos tangibles en relación con el total de activos (Gutiérrez, Morán y Posas, 2019).

$$
\text { Garantías }=\frac{\text { Activo Fijo Neto }}{\text { Total Activo }}
$$

Los autores nombrados denominan a esta variable como la tangibilidad. Según la teoría de trade off, se esperaría que esta variable tenga una relación directa con el nivel de endeudamiento, ya que el alto valor de los activos o el llamado colateral es apreciado por los acreedores. Autores como Titman y Wessels (1988); Serrasqueiro, Matías y Salsa (2016) demuestran una relación positiva entre la tangibilidad o las garantías que tiene la empresa y el nivel de endeudamiento.

Por el contrario, la teoría de jerarquía de preferencias propone una relación negativa de la variable garantía o tangibilidad con el nivel de endeudamiento, ya que esta teoría se enfoca en el uso de recursos internos para financiarse, y por tanto, el uso de la deuda como fuente de financiamiento es una decisión que queda en un segundo plano. Autores como, Huang y Song (2006); Padilla, Rivera y Ospina (2015), obtuvieron resultados en donde se evidencia la relación inversa entre las garantías o tangibilidad y el nivel de endeudamiento. 
Costo de la deuda: la estimación de este indicador se realiza de manera tradicional, siendo esta la relación existente entre los gastos financieros y el total de la deuda, con esto se busca evaluar la reducción de la tendencia a adquirir deuda ante su encarecimiento.

$$
\text { Costo de la Deuda }=\frac{\text { Gastos Financieros }}{\text { Total Pasivos }}
$$

Evidentemente, se esperaría que la relación entre el nivel de endeudamiento y el costo de la deuda sea negativa; esta relación subyace a partir de la función de inversión en la que se demuestra que existe una relación inversa entre inversión y la tasa de interés. Según Mochón (2009) la demanda de inversión viene dada por aumentos deseados o planeados por las empresas de capital físico e inventarios. Por tanto, a mayor costo de capital, menos incentivos tendrán los agentes económicos para financiar sus proyectos con deuda.

Oportunidades de crecimiento: es este un elemento para la valoración de los intangibles de la empresa y su capacidad de generación de valor. Su forma de cálculo se muestra a continuación:

$$
\text { Oportunidad de Crecimiento }=\frac{\text { Variación del ingreso }}{\text { Variación del Activo Total }}
$$

La teoría de trade off predice una relación negativa entre la variable oportunidades de crecimiento y el nivel de endeudamiento; según esta teoría, mientras mayores sean las oportunidades de crecimiento, mayor será el riesgo de quiebra y más elevados serán los costos de la agencia. Varias investigaciones han apoyado la teoría del trade off, como Serrasqueiro, Matías y Salsa (2016). 
Por su parte, la teoría de jerarquía de preferencias, supone una relación positiva entre oportunidades de crecimiento y el nivel de endeudamiento, ya que los recursos internos no son inagotables y para financiar grandes crecimientos se requiere una fuente alterna de recursos que será el endeudamiento. Acaravci (2015) concluye que existe una relación negativa entre el endeudamiento y las oportunidades de crecimiento.

Por otro lado, algunos autores como Serrasqueiro y Caetano (2014) y Titman y Wessels (1988) demuestran que no existe una relación estadísticamente significativa entre el nivel de endeudamiento y las oportunidades de crecimiento.

Reputación: esta variable será medida a través de la edad de la empresa, por eso es necesario hacer un análisis sobre empresas antiguas y activas a la fecha, pues, es un indicador de la seriedad y madurez de la empresa en el mercado además de una señal de prestigio.

$$
\text { Reputación }=\operatorname{Ln} \text { (años de existencia) }
$$

La variable reputación, según la teoría trade off, tiene una relación positiva con el nivel de endeudamiento mientras mayor sean sus años de existencia y, por tanto, su historial de crédito sea más amplio mayor capacidad tendrá la empresa de financiar sus inversiones con deuda.

Por el contrario, según la teoría de jerarquía financiera, la relación entre la reputación y el nivel de endeudamiento será negativa; pues, la empresa priorizará el financiamiento interno. Autores como Fernández y Murillo (2014) concluyen que existe una relación inversa entre el nivel de endeudamiento y la reputación de la empresa medida a través del logaritmo de los años de existencia. 
Liquidez: Mide la capacidad que tiene la empresa para poder cumplir con sus obligaciones de corto plazo (Besley y Brigham, 2016).

$$
\text { Liquidez }=\frac{\text { Activo Corriente }}{\text { Pasivo Corriente }}
$$

La teoría de trade off, predice una relación directa entre el nivel de liquidez y el nivel de endeudamiento de la empresa; es decir, empresas con alto nivel de liquidez tienen mayor capacidad de pago de las obligaciones.

La teoría de jerarquía de preferencias, en cambio, sugiere una relación negativa entre la liquidez y el nivel de endeudamiento de las empresas. Como se mencionó anteriormente, las empresas preferirán financiarse con utilidades retenidas antes que con deuda. Algunos autores refrendan esta teoría como: Serrasqueiro, Matías y Salsa (2016) y Gutiérrez, Morán y Posas (2019). 


\section{Resultados}

La variable dependiente del estudio es el nivel de endeudamiento medido por el ratio deuda total / activo total. Este sigue una distribución normal, con un valor medio de 0,58 y una desviación estándar de 0,19 que se valida con la prueba de Jarque-Bera. Debido a la existencia de empresas con patrimonio negativo (este tipo de empresas técnicamente se las consideraría en quiebra) el ratio máximo de endeudamiento es de 1,32. El ratio mínimo de endeudamiento de las empresas observadas fue de 0,0482 lo que evidencia que existen empresas que se apoyan más con financiamiento interno. La Figura 1 muestra la distribución de esta variable.

Figura 1. Distribución del ratio de endeudamiento de las grandes empresas manufactureras ecuatorianas, en el periodo $2008-2018$

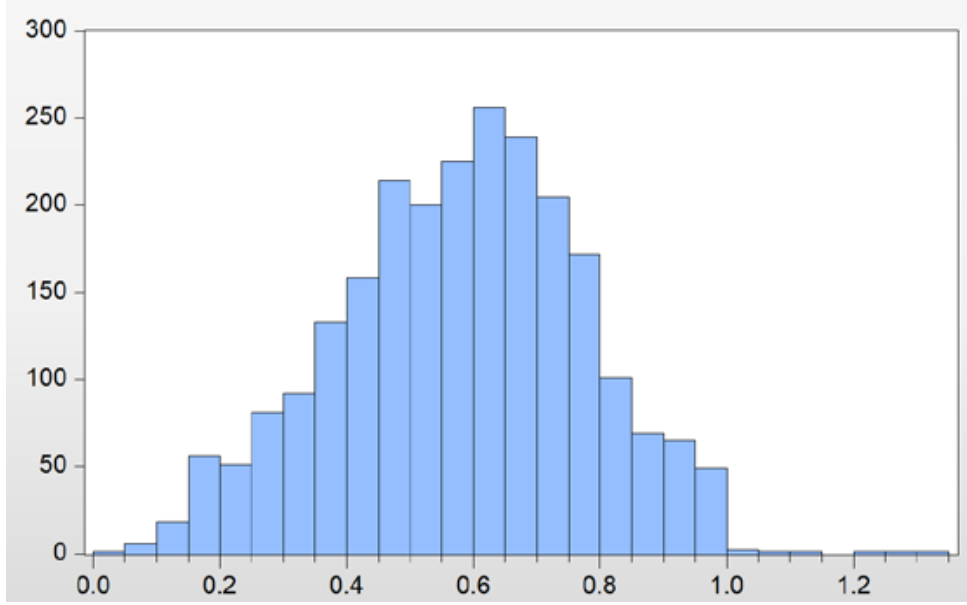

Series: RATIO_DE_ENDEUDAMIENTO Sample 20082018

Observations 2398

Mean $\quad 0.581041$

Median $\quad 0.593725$

Maximum $\quad 1.324574$

Minimum $\quad 0.048242$

Std. Dev. $\quad 0.193440$

Skewness $\quad-0.113212$

Kurtosis $\quad 2.719578$

Jarque-Bera 12.97961

Probability $\quad 0.001519$

Elaboración: Los autores. 
Al analizar la serie de tiempo del ratio de endeudamiento de las grandes empresas manufactureras, que se muestra en la Figura 2, se evidencia la caída del nivel de endeudamiento llegando a un 55,29\% a finales del año 2018. A medida que han pasado los años, las empresas han disminuido la participación de la deuda dentro su estructura de financiamiento, priorizando fuentes internas de financiamiento.

Figura 2. Nivel de endeudamiento de las grandes empresas manufactureras ecuatorianas, en el periodo 2008 - 2018

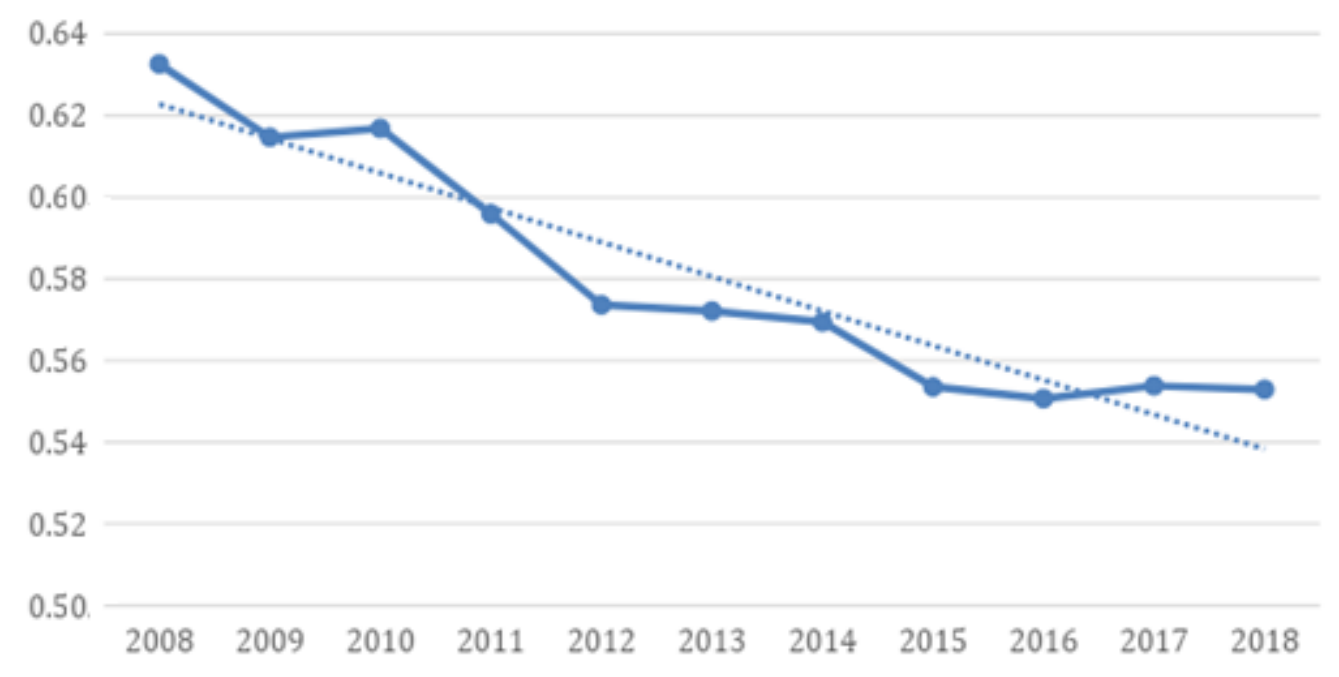

Elaboración: Los autores.

En la Figura 3 se observa la evolución de la serie de tiempo del logaritmo del activo total, que para el estudio se lo utiliza como una variable (proxy) que explica el tamaño de la empresa; en el transcurso del tiempo las empresas son más grandes y se puede afirmar que este incremento de los activos se financia en mayor proporción con recursos propios. 
Figura 3. Evolución del tamaño de las grandes empresas manufactureras ecuatorianas, medido a través del logaritmo del Activo Total, en el periodo $2008-2018$

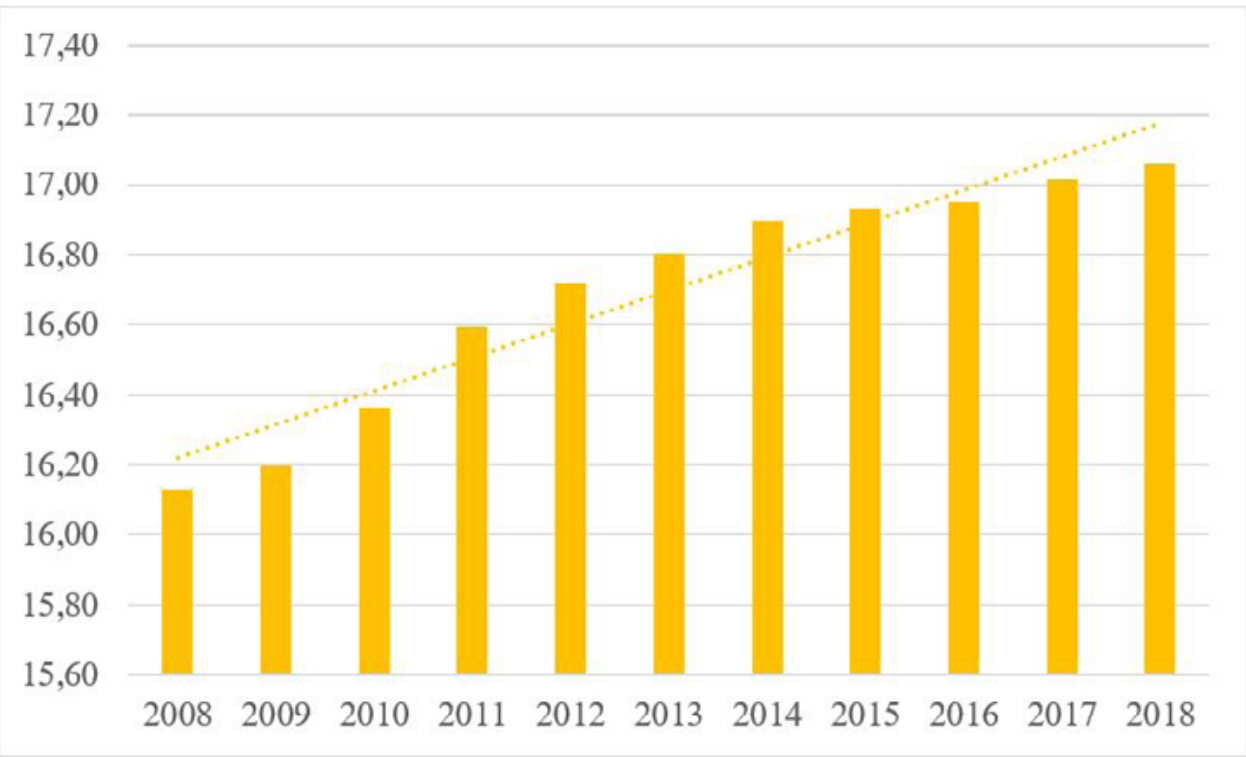

Elaboración: Los autores.

En cuanto a la evolución del ratio de liquidez, se observan dos períodos claramente marcados, el primero desde 2008 a 2014 en donde el ratio se mantuvo entre el 1,69 y 1,78 y un segundo período en donde este ratio experimenta un aumento hasta llegar al año 2016, con valor de 1,96 explicado por la aversión al riesgo de las empresas que decidieron dejar parte de sus fondos lo más líquidos posibles para hacer frente a la desaceleración que hubo de la economía ecuatoriana después del 2014, situación que se puede apreciar en la Figura 4. 
Figura 4. Evolución del ratio de liquidez de las grandes empresas manufactureras ecuatorianas, en el periodo 2008 - 2018

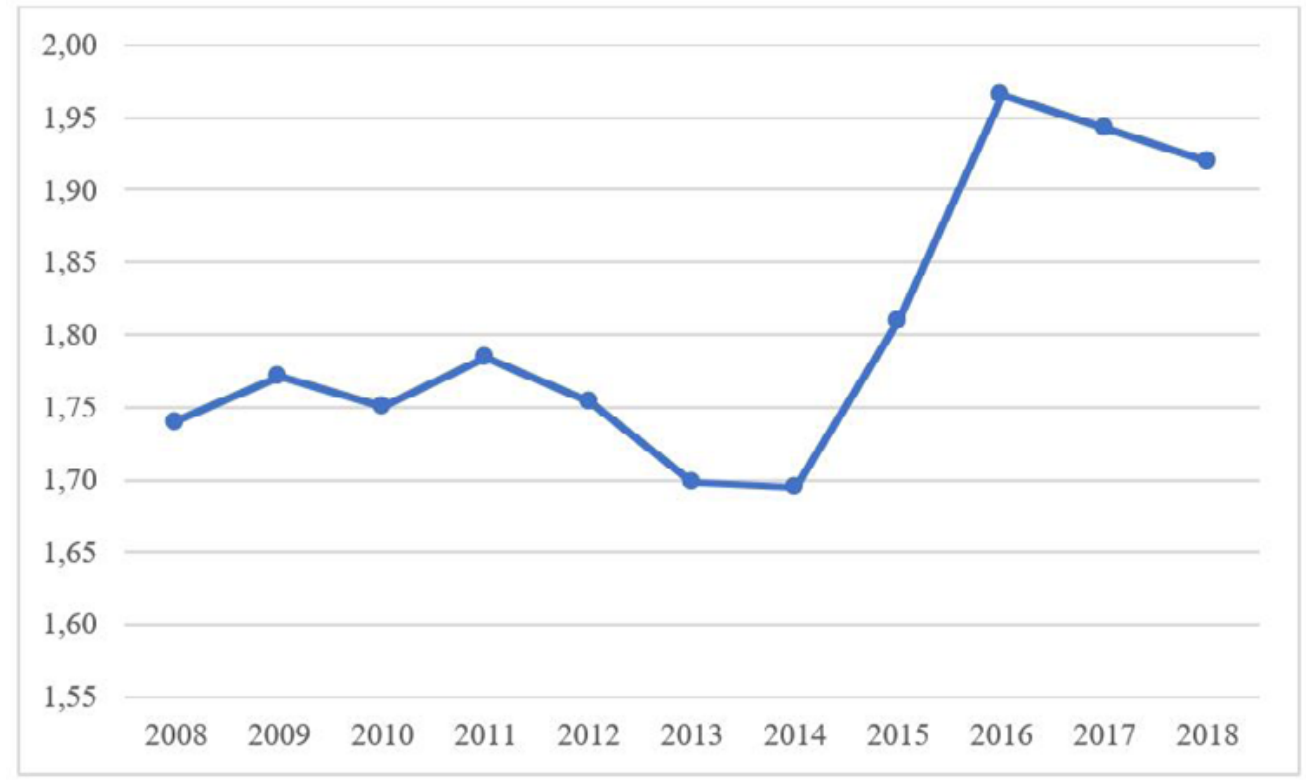

Elaboración: Los autores. 


\subsection{Estimación del \\ Modelo Econométrico}

Previo a la estimación de los parámetros se realizaron diferentes corridas econométricas con las variables propuestas, siendo la razón de endeudamiento la variable dependiente y el tamaño de la empresa, garantías, costo de la deuda, oportunidades de crecimiento, reputación y liquidez las diferentes variables independientes.

En el momento de realización del modelo se evidenciaron problemas de multicolinealidad entre el tamaño, tangibilidad y la variable oportunidades de crecimiento, por lo que se decidió incorporar en el modelo la variable tamaño, que engloba a las anteriores variables mencionadas. Para la realización del modelo de efectos aleatorios y fijos se utilizaron tres variables significativas:

- Tamaño

- Reputación

" Liquidez

En el Apéndice A se muestra la tabla con la estimación del modelo. A continuación, se presentan los resultados del modelo de efectos aleatorios:

Ratio de endeudamiento $=0,8604+0,0101$ (tamaño)-0,1195(reputación)0,0348 (liquidez) $+U_{i t}$

ee: $\quad(0,0720$

$(0,0052)$

$(0,0107)$

$(0,0020)$ 

$\mathrm{t}: \quad(1,4755)$
$(7,2737)$
$(-11,1246)$
$(-17,2111)$
$\mathrm{p}: \quad(0,000)$
$(0,0534)$
$(0,0000)$
$(0,0000)$
$R^{2}=0,1635$

Se realizó la prueba de Hausman para definir el método a ser utilizado en la estimación, los resultados que se visualizan en el Apéndice $B$ demuestran que se rechaza la hipótesis nula de que las estimaciones con el método de efectos fijos y aleatorios no tienen tanta diferencia, por lo que se utiliza el método de efectos fijos ya que el valor que presenta esta prueba es de 189,51 con un p valor menor al 5\%, lo que pone de manifiesto que la hipótesis de que los efectos individuales no están correlacionados con las variables regresoras debe ser rechazada con un nivel de confianza del 95\%; por tanto, el modelo de efectos aleatorios no es el adecuado y se procederá a trabajar con el modelo de efectos fijos.

En el Apéndice $C$ se muestra la tabla con la estimación del modelo. A continuación, se presentan los resultados del modelo de efectos fijos:

$$
\begin{aligned}
& \text { Ratio de endeudamiento }=0,1744+0,0531 \text { (tamaño) }-0,1316 \\
& \text { (reputación) }-0,0320 \text { (liquidez) }+U_{i t}
\end{aligned}
$$

$$
\begin{array}{lrrrr}
\text { ee: } & (0,1182) & (0,0073) & (-0,1320) & (-0,032) \\
\text { t: } & (1,4755) & (1,9324) & (-8,1195) & (-15,639) \\
\text { p: } & (0,1402) & (0,0000) & (0,0000) & (0,0000) \\
& & &
\end{array}
$$

El nuevo modelo utilizado demuestra que el tamaño de la empresa tiene una relación directa con el nivel de endeudamiento y existe una relación inversa con la liquidez de la empresa y su reputación. 
A mayor tamaño empresarial, mayor será su nivel de endeudamiento; mientras que, mejor reputación tenga la empresa (medida a través del logaritmo de los años de existencia) menor será el endeudamiento de las grandes empresas. De la misma manera, a mayor ratio de liquidez las empresas necesitarán menos financiamiento de terceros. Los coeficientes de cada una son: 0.0531 para el tamaño, -0.1316 para la reputación y -0.0320 para la liquidez.

La variación de las variables regresoras, explican en un $78.66 \%$ la variación del endeudamiento empresarial. 


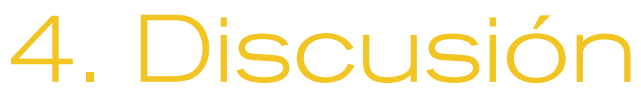

Este estudio ha explorado los determinantes del nivel de endeudamiento de las grandes empresas manufactureras ecuatorianas en el período comprendido entre el año 2008 y el año 2018, mediante un modelo de datos de panel. Para el análisis se tomaron en cuenta 218 empresas. Los resultados son muy similares a los presentados por Wadnipar y Cruz (2003); Gutiérrez, Moran y Posas (2019) y Montalván (2019), en donde variables como: tamaño de la empresa, rentabilidad, tangibilidad, liquidez y oportunidades de crecimiento explican en nivel de endeudamiento de las empresas analizadas. Los estudios mencionados anteriormente, concluyen que las empresas no basan sus decisiones de financiamiento en la posibilidad de encontrar una estructura óptima de capital sino que se basan en la teoría de jerarquía de preferencias, que es la que más se ajusta a la realidad del sector empresarial analizado.

En los estudios mencionados la variable que resultó ser estadísticamente significativa es el tamaño de la empresa, que tiene una relación directa con el nivel de endeudamiento $y$, a su vez, una relación inversa con la probabilidad de impago de empresas de menor tamaño, además que disponen de negocios más diversificados. Gutiérrez, Moran y Posas (2019) obtuvieron otras variables que explican el nivel de endeudamiento de las empresas analizadas como: rentabilidad, tangibilidad y liquidez, teniendo todas una relación inversa con el nivel de endeudamiento.

Los resultados del presente estudio, demuestran congruencia con los resultados de los estudios mencionados anteriormente, cuyas variables estadísticamente significativas son tres: 1) El tamaño empresarial, 2) la reputación de la empresa y 3) la liquidez. La primera, tiene una relación positiva y, las restantes, una relación negativa con el nivel de endeudamiento.

Gutiérrez, Moran y Posas (2019) concluyen que la razón por la que la liquidez, rentabilidad y tangibilidad tienen relación negativa y el tamaño tiene una relación positiva se da porque las empresas no deciden financiarse con deuda, por tanto, prefieren financiamiento con recursos 
propios. Así que, mientras más rentable y líquida sea la empresa, menos deuda requiere.

Con respecto a la tangibilidad las empresas con menores niveles de activos fijos tienen niveles más altos de endeudamiento. En ese caso se presume que estas empresas incurren en dos decisiones: 1) que otorguen activos corrientes como garantía y 2) que usen deuda de corto plazo como opción de financiamiento. 


\section{Conclusiones}

as empresas prefieren financiarse con recursos propios antes de contraer deuda con bancos o emitir documen-

tos. Más del $90 \%$ de las empresas son familiares, de tal manera, que los accionistas y administradores están emparentados. Esto deja poca cabida a inversionistas ajenos a la familia e, incluso, en la gran mayoría de casos el accionista actúa como gerente descartando la teoría de los costos de la agencia como explicación a las decisiones de financiamiento.

Aunque los costos financieros de la deuda son un factor importante para la toma de decisiones de endeudamiento, estadísticamente no resultaron ser significativos, por lo que se corrobora que la teoría de jerarquía de preferencias es la más adecuada para explicar el comportamiento de los empresarios a la hora de tomar decisiones sobre el tipo de la fuente de financiamiento a escoger.

Este estudio tiene dos limitaciones: 1) no diferencia entre deuda de corto y largo plazo, sino que se trabaja con la deuda total y 2) se estableció un modelo único de datos de panel para las empresas grandes del sector manufacturero ecuatoriano, sin diferenciar la singularidad de las industrias analizadas.

Con las limitaciones mencionadas se propone para futuros trabajos el estudiar los determinantes del nivel de endeudamiento empresarial de corto plazo y largo plazo. Además, se debe estudiar la singularidad de las diferentes industrias del sector manufacturero, diferenciándolas por subsector y tamaño. 


\section{Referencias Bibliográficas}

Acaravci, S. (2015). The Determinants of Capital Structure: Evidence from the Turkish Manufacturing Sector. International Journal of Economics and Financial Issues, 5(1), 158-171. https://doi.org/10.1108/ AJEMS-11-2012-0072

Acebedo, M., Alútiz, A., y Ruiz, F. (2012). Factores determinantes de la estructura de capital de las empresas españolas. Tribuna de Economía, 868(1), 155-171.

Baronio, A., y Vianco, A. (2014). Datos de Panel. Guía para el uso de Eviews. http://www.econometricos.com.ar/wp-content/ uploads/2012/11/datos-de-panel.pdf

Besley, S., y Brigham, E. (2016). Fundamentos de Administración Financiera (Decimacuarta Ediciones) Cengage Learning Editores.

Contreras, N., y Díaz, E. (2015). Estructura financiera y rentabilidad: origen, teorías y definiciones. Revista de Investigación Valor Contable, 2(1), 35-44. https://doi.org/10.17162/rivc.v2i1.824

Brealey, R., Myres, S., y Allen, F. (2015). Principios de Finanzas Corporativas (Undécima Edición). McGraw-Hill Global Education Holdings.

De Andrés, P., de la Fuente, G., y San Martín, P. (2018). Capital structure decisions: What Spanish CFOs think. Academia Revista Latinoamericana de Administración, 31(2), 306-325. https://doi. org/10.1108/ARLA-10-2016-0267

Durand, D. (1952). Costs of Debt and Equity Funds for Business: Trends and Problems of Measurement. National Bureau of Economic Research.

Fernández, H., y Murillo, S. (2014). Un análisis con datos de panel de los factores explicativos del nivel de endeudamiento de las empresas colombianas en la Universidad de Medellín. En F. Isaza (Ed.), Finanzas, modelación y estrategias (p. 57). Sello Editorial. 
Greene, W. (1998). Análisis Econométrico (Tercera Edición). Prentice Hall.

Gujarati, D., y Porter, D. (2010). Econometría (Quinta Edición). McGrawHill/Interamericana.

Gutiérrez, H., Morán, C., y Posas, R. (2018). Determinantes de la estructura de capital: un estudio empírico del sector manufacturero en Ecuador. Contaduría y Administración, 64(2), 1-19. https://doi.org/10.22201/ fca.24488410e.2018.1848

Huang, G., y Song, F. (2006). The determinants of capital structure: Evidence from China. China Economic Review, 17(1), 14-36. https:// doi.org/10.1016/j.chieco.2005.02.007

Jensen, M. (1986). Agency costs of free cash flow, corporate finance, and takeovers. The American Economic Review, 76(2), 323-329. https:// doi.org/10.1017/cbo9780511609435.005

Kim, W., y Sorensen, E. (1986). Evidence on the Impact of the Agency Costs of Debt on Corporate Debt Policy. The Journal of Financial and Quantitative Analysis, 21(2), 131-144. https://doi. org/10.2307/2330733

Mayorga, M., y Muñoz, E. (2000). La técnica de datos de panel. Una guía para su uso e interpretación. http://www.seti.chubut.gov.ar/Publico/ PDF/Mayorga y Muñoz Técnica de datos de panel.pdf

Medina, A., Salinas, J., Ochoa, L., y Molina, C. (2012). La estructura financiera de las empresas manufactureras colombianas, una mirada econométrica, macroeconómica y financiera. Perfil de Coyuntura Económica, 0(20), 155-176

Miller, M. (1977). Debt and taxes. The Journal of Finance, 32(2), 261-275.

Mochón, F. (2009). Economía, teoría y política (Sexta Edición). McGrawHill/Interamericana de España.

Modigliani, F., y Miller, M. (1958). The Cost of Capital, Corporation Finance and the Theory of Investment. The American Economic Review, 48(3), 261-297. https://doi.org/10.1136/bmj.2.3594.952 
Modigliani, F., y Miller, M. (1963). Corporate Income Taxes and the Cost of Capital: A Correction. The American Economic Review, 53(3), 433-443. https://doi.org/10.2307/1809167

Mongrut, S., Fuenzalida, D., Pezo, G., y Zdenko, T. (2010). Explorando teorías de estructura de capital en latinoamérica. Cuadernos de Administración, 23(41), 163-184. http://www.scielo.org.co/pdf/ $\mathrm{cadm} / \mathrm{v} 23 \mathrm{n} 41 / \mathrm{v} 23 \mathrm{n} 41 \mathrm{a} 08 . \mathrm{pdf}$

Montalván, J. (2019). Determinantes de la Estructura de Capital: Un Análisis de las Pymes Ecuatorianas con Financiamiento en el Mercado de Valores. X-Pedientes Económicos, 3 (7), 57-75.

Myres, S. (1984). The Capital Structure Puzzle. National Bureau of Exonomic Research (Issue 1393). https://doi.org/10.1111/j.1540-6261.1984. tb03646.x

Padilla, A., Rivera, J., y Ospina, J. (2015). Determinantes de la estructura de capital de las mipyes del sector real participantes del premio innova 2007-2011. Revista Finanzas y Política Económica, 7(2), 359-380. https://doi.org/10.14718/revfinanzpolitecon.2015.7.2.8

Paredes, A., Ángeles, G., y Flores, M. (2016). Determinants of leverage in mining companies, empirical evidence for Latin American countries. Contaduría y Administración, 61(1), 26-40. https://doi.org/10.1016/j. cya.2015.09.010

Rivera, J. (2002). Teoría sobre la estructura de capital. Estudios Gerenciales, 18(84), 31-59. https://doi.org/10.18046/j.estger.2002.93

Schwartz, E. (1959). Theory of the Capital Structure of the Firm. The Journal of Finance, 14(1), 18-39. https://doi.org/10.1111/j.1540-6261.1959. tb00483.x

Serrasqueiro, Z., y Caetano, A. (2015). Trade-Off Theory versus Pecking Order Theory: capital structure decisions in a peripheral region of Portugal. Journal of Business Economics and Management, 16(2), 445-466. https://doi.org/10.3846/16111699.2012.744344 
Serrasqueiro, Z., Matias, F., y Salsa, L. (2016). Determinants of capital structure: New evidence from Portuguese small firms. Dos Algarves: A Multidisciplinary e-Journal, 28, 13-28. https://doi.org/10.18089/ damej.2016.28.1.2

Stulz, R. (1990). Managerial discretion and optimal financing policies. Journal of Financial Economics, 26(1), 3-27. https://doi. org/10.1016/0304-405X(90)90011-N

Superintendencia de Compañías, Valores y Seguros. (2020). Portal de información. https://appscvsmovil.supercias.gob.ec/ portallnformacion/index.zu

Titman, S., y Wessels, R. (1988). The Determinants of Capital Structure Choice. The Journal of Finance, 43(1), 1-19. https://doi. org/10.1111/j.1540-6261.1988.tb02585.x

Tresierra, Á. (2008). Comportamiento de la estructura financiera en un grupo de empresas españolas previa a la participación del capital riesgo (Issue May). https://doi.org/1698-8183

Wadnipar, S., y Cruz, J. (2008). Determinación de la estructura de capital de las empresas colombianas. Revista Soluciones de Postgrado EIA, $1,23-44$.

Wooldridge, J. (2010). Introducción a la econometría, un enfoque moderno (4th ed.). Cengage Learning.

Zambrano, S., y Acuña, G. (2011). Estructura de Capital. Evolución Teórica. Criterio Libre, 9(15), 81-102. http://search.proquest.com/ docview/1010350445?accountid=17214 


\section{APÉNDICE A}

Dependent Variable: RATIO_DE_ENDEUDAMIENTO

Method: Panel EGLS (Two-way random effects)

Date: $05 / 05 / 20$ Time: $20: 37$

Sample: 20082018

Periods included: 11

Cross-sections included: 218

Total panel (balanced) observations: 2398

Swamy and Arora estimator of component variances

\begin{tabular}{|c|c|c|c|c|}
\hline Variable & Coefficient & Std. Error & t-Statistic & Prob. \\
\hline TAMANO & 0.010114 & 0.005234 & 1.932434 & 0.0534 \\
\hline REPUTACION & -0.119596 & 0.010751 & -11.12469 & 0.0000 \\
\hline LIQUIDEZ & -0.034890 & 0.002027 & -17.21115 & 0.0000 \\
\hline $\mathrm{C}$ & 0.860493 & 0.072080 & 11.93797 & 0.0000 \\
\hline \multicolumn{5}{|c|}{ Effects Specification } \\
\hline & & & S.D. & Rho \\
\hline Cross-section random & & & 0.131827 & 0.6628 \\
\hline Period random & & & 0.002815 & 0.0003 \\
\hline Idiosyncratic random & & & 0.093981 & 0.3369 \\
\hline
\end{tabular}

Weighted Statistics

\begin{tabular}{llll}
\hline \hline R-squared & 0.169350 & Mean dependent var & 0.121582 \\
Adjusted R-squared & 0.168309 & S.D. dependent var & 0.105517 \\
S.E. of regression & 0.096229 & Sum squared resid & 22.16827 \\
F-statistic & 162.6938 & Durbin-Watson stat & 0.754381 \\
Prob(F-statistic) & 0.000000 & & \\
\hline \hline
\end{tabular}

Unweighted Statistics

\begin{tabular}{llll}
\hline \hline R-squared & 0.180216 & Mean dependent var & 0.581041 \\
Sum squared resid & 73.52949 & Durbin-Watson stat & 0.227878
\end{tabular}




\section{APÉNDICE B}

Correlated Random Effects - Hausman Test

Equation: Untitled

Test cross-section and period random effects

\begin{tabular}{lrrr}
\hline \hline Test Summary & Chi-Sq. Statistic & Chi-Sq. d.f. & Prob. \\
\hline \hline Cross-section random & 189.513960 & 3 & 0.0000 \\
Period random & 0.000000 & 3 & 1.0000 \\
Cross-section and period random & 184.018407 & 3 & 0.0000 \\
\hline \hline
\end{tabular}

${ }^{\text {* }}$ Period test variance is invalid. Hausman statistic set to zero.

Cross-section random effects test comparisons:

\begin{tabular}{crrrr} 
Variable & Fixed & Random & $\operatorname{Var}$ (Diff.) & Prob. \\
\hline \hline TAMANO & 0.030026 & 0.010114 & 0.000015 & 0.0000 \\
REPUTACION & -0.176042 & -0.119596 & 0.000069 & 0.0000 \\
LIQUIDEZ & -0.032035 & -0.034890 & 0.000000 & 0.0000 \\
\hline \hline
\end{tabular}

\section{APÉNDICE C}

Dependent Variable: RATIO_DE_ENDEUDAMIENTO

Method: Panel Least Squares

Date: $05 / 05 / 20$ Time: $20: 14$

Sample: 20082018

Periods included: 11

Cross-sections included: 218

Total panel (balanced) observations: 2398

\begin{tabular}{crrrr}
\hline \hline Variable & Coefficient & Std. Error & t-Statistic & Prob. \\
\hline \hline TAMANO & 0.053182 & 0.007312 & 7.273751 & 0.0000 \\
REPUTACION & -0.131619 & 0.016210 & -8.119517 & 0.0000 \\
LIQUIDEZ & -0.032000 & 0.002046 & -15.63915 & 0.0000 \\
C & 0.174441 & 0.118223 & 1.475529 & 0.1402 \\
\hline \hline
\end{tabular}

Effects Specification

Cross-section fixed (dummy variables)

Period fixed (dummy variables)

\begin{tabular}{lllr}
\hline \hline R-squared & 0.786609 & Mean dependent var & 0.581041 \\
Adjusted R-squared & 0.763960 & S.D. dependent var & 0.193440 \\
S.E. of regression & 0.093981 & Akaike info criterion & -1.800082 \\
Sum squared resid & 19.13982 & Schwarz criterion & -1.243062 \\
Log likelihood & 2389.298 & Hannan-Quinn criter. & -1.597429 \\
F-statistic & 34.73074 & Durbin-Watson stat & 0.820475 \\
Prob(F-statistic) & 0.000000 & & \\
\end{tabular}

